\title{
NECESSARY CONDITIONS FOR WEAK LOWER SEMICONTINUITY ON DOMAINS WITH INFINITE MEASURE
}

\author{
STEFAN KRÖMER ${ }^{1}$
}

\begin{abstract}
We derive sharp necessary conditions for weak sequential lower semicontinuity of integral functionals on Sobolev spaces, with an integrand which only depends on the gradient of a scalar field over a domain in $\mathbb{R}^{N}$. An emphasis is put on domains with infinite measure, and the integrand is allowed to assume the value $+\infty$.
\end{abstract}

Mathematics Subject Classification. 49J45.

Received August 8, 2008. Revised December 23, 2008.

Published online April 21, 2009.

\section{INTRODUCTION}

We consider functionals of the type

$$
G(u):=\int_{\Omega} g(\nabla u) \mathrm{d} x
$$

where $u \in L_{\sim}^{1, p}(\Omega)$ or $u \in\left(L^{1, p} \cap L^{q}\right)(\Omega)$. A definition of these spaces is given below. Here and throughout the rest of the paper, all functions are real-valued (except gradients, which take values in $\mathbb{R}^{N}$ ). Moreover, throughout we assume that

$$
\begin{aligned}
& \Omega \subset \mathbb{R}^{N} \text { is nonempty, open and connected, } 1 \leq p<\infty \text { and } 1 \leq q<\infty, \\
& g: \mathbb{R}^{N} \rightarrow \mathbb{R} \cup\{+\infty\} \text { is a Borel function, and } \\
& g(\xi) \geq-C|\xi|^{p} \text { for every } \xi \in \mathbb{R}^{N},
\end{aligned}
$$

with a constant $C>0$, which makes sure that $G$ as a map into $\mathbb{R} \cup\{+\infty\}$ is well defined on each of the two mentioned spaces of admissible functions. Although the results below do not require any further assumptions on $\Omega$ (unless explicitly stated otherwise), our main focus is on domains with infinite measure.

A natural space of admissible functions for $G$ is

$$
L^{1, p}(\Omega):=\left\{\left.u \in W_{\mathrm{loc}}^{1, p}(\Omega)\left|\int_{\Omega}\right| \nabla u\right|^{p} \mathrm{~d} x<\infty\right\},
$$

Keywords and phrases. Scalar integral functionals, weak lower semicontinuity, necessary conditions.

1 Department of Mathematical Sciences, Carnegie Mellon University, Pittsburgh, PA 15213-3890, USA.

kroemers@andrew.cmu.edu 
equipped with the seminorm

$$
\|u\|_{L^{1, p}(\Omega)}:=\left(\int_{\Omega}|\nabla u|^{p} \mathrm{~d} x\right)^{\frac{1}{p}}, \text { where }|\nabla u|:=\left(\sum_{i=1}^{N}\left(\partial_{i} u\right)^{2}\right)^{\frac{1}{2}} .
$$

This becomes a norm if we identify functions whose gradients coincide almost everywhere. Thus we are looking at the space

$$
L_{\sim}^{1, p}(\Omega):=\left\{[u] \mid u \in L^{1, p}(\Omega)\right\},
$$

where $[u]=\left\{\tilde{u} \in L^{1, p}(\Omega) \mid \nabla u=\nabla \tilde{u}\right.$ a.e. $\}$. This is a Banach space with the norm $\|[u]\|_{L_{\sim}^{1, p}(\Omega)}:=\|u\|_{L^{1, p}(\Omega)}$, and weak convergence of a sequence $\left(u_{n}\right)$ in $L_{\sim}^{1, p}(\Omega)$ is equivalent to the weak convergence of $\left(\nabla u_{n}\right)$ in $L^{p}\left(\Omega ; \mathbb{R}^{N}\right)$ (see e.g. [5]). As an alternative setting, we also discuss the space $\left(L^{1, p} \cap L^{q}\right)(\Omega)$ for $1<q<\infty$, which is a Banach space with respect to the norm

$$
\|u\|_{\left(L^{1, p} \cap L^{q}\right)(\Omega)}:=\left(\int_{\Omega}|\nabla u|^{p} \mathrm{~d} x\right)^{\frac{1}{p}}+\left(\int_{\Omega}|u|^{q} \mathrm{~d} x\right)^{\frac{1}{q}} .
$$

The special case $q=p$ includes the classical Sobolev space $W^{1, p}(\Omega)$. On domains with infinite measure, $L^{1, p} \cap L^{q}$ consist of functions $u$ which in some sense vanish at infinity as integrability of $|u|^{q}$ is required. Moreover, note that if $p<N$, we have a natural identification $L_{\sim}^{1, p}(\Omega)=\left(L^{1, p} \cap L^{p^{*}}\right)(\Omega)$ with $p^{*}:=\frac{p N}{N-p}$ for a large class of domains $\Omega$ with infinite measure and suitable geometrical properties, including the whole space. For details, we refer to the appendix.

As lower semicontinuity of functionals with respect to suitable weak topologies is the cornerstone of the so-called direct methods in the calculus of variations, there is a rich literature providing conditions of the integrand $g$ which ensure this property of the corresponding functional $G$. In our simple setting, $G$ is wslsc ${ }^{2}$ in $L_{\sim}^{1, p}(\Omega)$ provided that $g$ is convex, $\operatorname{lsc}^{3}$ in $\mathbb{R}^{N}$ and nonnegative (for $p>1$ ). Of course, this is a rather standard result, which we recall in Section 2. It is natural to ask whether these sufficient conditions are sharp, and thus we are interested in finding conditions on $g$ which are necessary for wslsc ${ }^{4}$ of $G$, much in the spirit of [2] but now for functionals depending on the gradient. Also note that the precise knowledge of conditions on the integrand which are necessary and sufficient for wslsc is crucial for determining the representation of a relaxed functional (the largest wslsc functional below a given functional which is not wslsc). If $\Omega$ has finite measure, necessary conditions for wslsc of $G$ are well known even for more general functionals, although usually $g$ is also assumed to be real-valued, or even to satisfy a $p$-growth condition $([1,3], e . g$.$) . The case of a domain with infinite measure$ together with an integrand $g$ which is allowed to assume the value $+\infty$ has been open so far.

The main results of this note, Theorem 4.1 (for $G$ defined on $L_{\sim}^{1, p}$ ) and Theorem 4.2 (for $G$ defined on $\left.L^{1, p} \cap L^{q}\right)$ in Section 4 , state that the aforementioned sufficient conditions are indeed essentially necessary if $\Omega$ has infinite measure. The word "essentially" is included here to signify that - somewhat surprisingly - there are counterexamples for functionals which are trivial in the sense of Definition 3.1, as we shall see in Section 3. The main task in the proof of our main results hence is to make use of suitable conditions which rule out trivial functionals to avoid this problem. On a technical level, complications in the proof come from the fact that on a domain with infinite measure, functions with merely bounded gradient in general do not have $p$-integrable gradient, which prevents the use of the simple constructions one could utilize to deduce properties of $g$ from the ones of $G$ for functionals on domains with finite measure, in analogy of the corresponding results for functionals not involving derivatives in $L^{p}$ spaces, presented in [2]. Thus, we have to rely on a known admissible function $u$ with $G(u)<\infty$ to correct the behavior of explicitly constructed functions in the outer part of $\Omega$ in such a way

\footnotetext{
${ }^{2}$ Weakly sequentially lower semicontinuous, i.e., $\liminf G\left(w_{n}\right) \geq G(w)$ whenever $w_{n} \rightarrow w$ weakly.

${ }^{3}$ (Sequentially) lower semicontinuous, i.e., $\liminf g\left(\xi_{n}\right) \geq g(\xi)$ whenever $\xi_{n} \rightarrow \xi$.

${ }^{4}$ Weak sequential lower semicontinuity.
} 
that weak differentiability is preserved and $G$ stays finite. We are able to do this by exploiting some of the properties of the "pyramid" of Lemma 3.5, on which our construction is based.

\section{SuFFICIENT CONDITIONS}

For comparison with our main result in Section 4, we now recall the standard conditions implying wslsc of $G$. In our simple setting, they go back to the following well-known abstract result.

Theorem 2.1 (e.g. [1] or [3]). Let $X$ be a Banach space and let $F: X \rightarrow \mathbb{R} \cup\{+\infty\}$ be convex and strongly $l s c^{5}$. Then $F$ is wslsc on $X$.

In our framework, this yields:

Corollary 2.2. Let $p \in[1, \infty)$, let $X=L_{\sim}^{1, p}(\Omega)$ or $X=\left(L^{1, p} \cap L^{q}\right)(\Omega)$, respectively, and suppose that $g$ is convex, lsc and nonnegative. Then $G$ is wslsc on $X$.

Proof. Since convexity of $g$ immediately implies convexity of $G$, it suffices to show that $G$ is strongly lsc, and the latter is a consequence of Fatou's lemma.

In the case $p=1$, a slight improvement is possible.

Corollary 2.3. Let $X=L_{\sim}^{1,1}(\Omega)$ or $X=\left(L^{1,1} \cap L^{q}\right)(\Omega)$, respectively. Moreover, suppose that $g$ is convex and lsc, and $g(\xi) \geq \nu \cdot \xi$ for a constant $\nu \in \mathbb{R}^{N}$. Then $G$ is wslsc on $X$.

Proof. By Corollary 2.2, we get wslsc of $\tilde{G}(u):=G(u)-f(u)$, where $f(u):=\int_{\Omega} \nu \cdot \nabla u \mathrm{~d} x$. Since $f$ is a continuous linear functional on $X$, this implies wslsc of $G$.

Of course, these results are not sharp in general, and the extent to which the assumptions can be relaxed in fact strongly depends on the domain. For domains with finite measure, it is well known that besides (1.4), no additional lower bounds on $g$ are needed (the convexity of $g$ and (1.4) still imply that $g(\xi) \geq \nu \cdot \xi-C$ for constants $\nu \in \mathbb{R}^{N}$ and $C \in \mathbb{R}$, and for domains with finite measure, this suffices even for $p>1$ ). Neither are the sufficient conditions given in the corollaries above sharp in general for domains with infinite measure. However, this is entirely due to the fact that the value $+\infty$ is allowed for $g$, which may cause the existence of trivial functionals with nontrivial Lagrangian $g$ as we shall see next.

\section{TRIVIAL FUNCTIONALS}

Definition 3.1. A function $F: D \rightarrow \mathbb{R} \cup\{+\infty\}$ is called trivial, if it is finite at at most finitely many points in $D$.

The use of the term "trivial" here is motivated by the fact that such a functional is automatically lsc, no matter what topology is being used. If $\Omega$ has finite measure, $G$ defined on $L_{\sim}^{1, p}(\Omega)$ is trivial if and only if $g$ is finite at at most one point and $G$ defined on $\left(L^{1, p} \cap L^{q}\right)(\Omega)$ is trivial if and only if $g \equiv+\infty$. For domains with infinite measure, the picture is more complicated, and this has immediate consequences concerning necessary conditions for wslsc of $G$, as the following examples illustrate.

Example 3.2. Consider $\Omega=\mathbb{R}^{N}$ with $N \geq 2$, and

$$
g(\xi):= \begin{cases}0 & \text { if } \xi=0 \\ 2 & \text { if } \xi=e(\text { a fixed unit vector }) \\ 1 & \text { if } \xi=\left(1+\frac{1}{n}\right) e \text { for an } n \in \mathbb{N} \\ +\infty & \text { elsewhere }\end{cases}
$$

In this case, $G: L_{\sim}^{1, p}(\Omega) \rightarrow(-\infty, \infty]$ is finite only at [0]. In particular, $G$ is (strongly) lsc and wslsc in $L_{\sim}^{1, p}$, despite the fact that $g$ is neither lsc nor convex.

\footnotetext{
${ }^{5}$ (Sequentially) lower semicontinuous with respect to the strong (norm) convergence in $X$.
} 
Proof. Consider $[u] \in L_{\sim}^{1, p}\left(\mathbb{R}^{N}\right)$ with $G(u)<\infty$. For $E:=\mathbb{R}^{N} \backslash\{\nabla u=0\}$ we have $|E|<\infty$ and $\nabla u(x) \in\{\alpha e \mid$ $\alpha \geq 1\}$ for a.e. $x \in E$. In particular, $\nabla u$ is always parallel to $e$, whence $u$ is constant on the hyperplanes

$$
H_{t}:=\left\{x \in \mathbb{R}^{N} \mid x \cdot e=t\right\}
$$

for a.e. $t \in \mathbb{R}$, and thus $\nabla u \in\{\alpha e \mid \alpha \geq 1\}$ a.e. on $H_{t}$, for a.e. $t$ such that $\mathcal{H}^{N-1}\left(H_{t} \cap E\right)$ (the $N$-1-dimensional measure of $H_{t} \cap E$ ) is positive. Hence,

$$
\infty>\int_{\Omega} g(\nabla u) \geq \int_{S} g(\nabla u) \geq|S| \text { for the strip } S:=\bigcup_{\mathcal{H}^{N-1}\left(H_{t} \cap E\right)>0} H_{t} .
$$

But by Fubini's theorem, $|S|<\infty$ if and only if $|E|=0$.

Note that the above example strongly hinges on the geometry of the domain, here the whole space. If the class of admissible functions is subject to a built-in decay at infinity, such as in $W^{1, p}\left(\mathbb{R}^{N}\right)$ or $L_{\sim}^{1, p}\left(\mathbb{R}^{N}\right)=$ $L^{1, p}\left(\mathbb{R}^{N}\right) \cap L^{p^{*}}\left(\mathbb{R}^{N}\right)$ for $p<N(c f$. Thm. A.2), even more general constructions are possible.

Example 3.3. For $N \geq 2$ consider the convex cone

$$
\Omega=\Lambda:=\left\{x \in \mathbb{R}^{N}|x \cdot e>\gamma| x \mid\right\}, \text { with } e \in S^{N-1} \text { and } \gamma \in[0,1) \text { fixed, }
$$

and assume that there are two unit vectors $\mu, \nu \in \bar{\Lambda}$ such that $\{g<\infty\} \subset \bar{H}_{\mu} \cap \bar{H}_{-\nu}$, where $\bar{H}_{\xi}:=\left\{y \in \mathbb{R}^{N} \mid\right.$ $y \cdot \xi \geq 0\}$. Then $G: L^{1, p} \cap L^{q}(\Lambda) \rightarrow \mathbb{R} \cup\{\infty\}$ is finite at most at $u=0$. In particular, it is strongly lsc and wslsc, despite the fact that $g$ does not have to be lsc or convex.

Proof. Consider $u \in L^{1, p}\left(\mathbb{R}^{N}\right) \subset L^{q}\left(\mathbb{R}^{N}\right)$ with $G(u)<\infty$. As a consequence, $\nabla u \in \bar{H}_{\mu} \cap \bar{H}_{-\nu}$ a.e., which implies that for a.e. $x_{0} \in \Lambda, v:(0, \infty) \rightarrow \mathbb{R}, v(t):=u\left(x_{0}+t \mu\right)$, is nondecreasing, and $w:(0, \infty) \rightarrow \mathbb{R}$, $w(t):=u\left(x_{0}+t \nu\right)$, is nonincreasing. Moreover, since $v \in L^{q}((0, \infty))$ for a.e. $x_{0} \in \Omega$, we infer that $v(t) \rightarrow 0$ as $t \rightarrow+\infty$, for any such $x_{0}$. As a consequence, $v \leq 0$ for a.e. $x_{0}$, whence $u \leq 0$ a.e. Repeating the argument with $w$ instead of $v$, we also get $u \geq 0$ a.e.

Example 3.4. Consider $\Omega=\mathbb{R}^{N}$ and assume that $g(\xi)=+\infty$ for any $\xi \in H$, where $H \subset \mathbb{R}^{N}$ is a given open half-space. Then $G: L^{1, p} \cap L^{q}\left(\mathbb{R}^{N}\right) \rightarrow \mathbb{R} \cup\{\infty\}$ is finite at most at $u=0$. In particular, it is strongly lsc and wslsc, despite the fact that $g$ does not have to be lsc or convex.

Proof. Consider $u \in L^{1, p}\left(\mathbb{R}^{N}\right) \subset L^{q}\left(\mathbb{R}^{N}\right)$ with $G(u)<\infty$, and let $e$ denote the unit vector in $\mathbb{R}^{N}$ such that $H=\left\{y \in \mathbb{R}^{N} \mid e \cdot y>0\right\}$. Arguing as in the proof of Example 3.3, we get that for a.e. $x_{0} \in \mathbb{R}^{N}, v: \mathbb{R} \rightarrow \mathbb{R}$, $v(t):=u\left(x_{0}+t e\right)$, is nonincreasing, and $v(t) \rightarrow 0$ as $t \rightarrow \pm \infty$. Hence $v=0$ for a.e. $x_{0}$, and $u=0$ a.e., accordingly.

Example 3.4 works whenever 0 is not contained in the interior of the convex hull of the set $\{g<\infty\}:=\{\xi \in$ $\left.\mathbb{R}^{N} \mid g(\xi)<\infty\right\}$. If this behavior is ruled out, we have the following construction, which will be at the heart of the proofs of Section 4 :

Lemma 3.5. Let $F \subset \mathbb{R}^{N}$ be a finite set such that $0 \in(\operatorname{co} F)^{\circ}$, the interior of the convex hull of $F$ (in particular, $F$ has at least $N+1$ elements). Then the piecewise affine "pyramid"

$$
P(x):=\min \left\{\eta \cdot x=\sum_{i=1}^{N} \eta_{i} x_{i} \mid \eta \in F\right\}, x \in \mathbb{R}^{N},
$$

is a continuous function which satisfies $P \in W_{\text {loc }}^{1, \infty}\left(\mathbb{R}^{N}\right), \nabla P \in F$ a.e., $P(0)=0$, and $P(x) \leq-a|x|$ for $x \in \mathbb{R}^{N}$, with the constant $a:=-\min \{P(x)|| x \mid=1\}>0$. 
Remark 3.6. If $0 \in(\operatorname{co} E)^{\circ}$, where $E \subset \mathbb{R}^{N}$ is an infinite set, it is always possible to select a subset $F \subset E$ with at most $2 N$ elements such that $0 \in(\operatorname{co} F)^{\circ}$, cf. [4].

Remark 3.7. The crucial property of $P$ for our arguments below is its uniform negative linear growth as $|x| \rightarrow \infty$. To be more precise, we essentially need that for a suitable finite subset $F \subset\{g<\infty\}$, the associated $P$ has the property that

$$
P(x) \leq-a|x|+C \text { for every } x \in \Omega \text { with constants } a>0 \text { and } C \in \mathbb{R} \text {. }
$$

Alternatively, with obvious changes in the proofs, we could work under the assumption that

$$
\begin{array}{r}
\tilde{P}(x) \geq a|x|-C \text { for every } x \in \Omega \text { with constants } a>0 \text { and } C \in \mathbb{R}, \\
\text { where } \tilde{P}(y):=\max \{\eta \cdot y \mid \eta \in F\} \text { for } y \in \mathbb{R}^{N} .
\end{array}
$$

Here, note that both conditions are invariant with respect to translations of $\Omega$ up to a possible change in the constant $C$, and $\tilde{P}(x)=-P(-x)$, which means that (3.1) holds for $\Omega$ if and only if (3.2) holds for $\tilde{\Omega}:=-\Omega$ instead of $\Omega$. Technically, (3.1) (or its variant (3.2)) is important for us because it allows us to build a piece of $P$ into a given admissible function for $G$ : Whenever $u$ is an admissible function with $G(u)<\infty$ such that $u$ has "sublinear growth" in the sense of (3.3) below, (3.1) implies that $v:=\max \{P+h, u\}$ (with arbitrary fixed $h \in \mathbb{R}$, the choice of which can be used to make sure that $v \neq u$ ) differs from $u$ only on a set of finite measure, whence $v$ is an admissible function for $G$ and $G(v)<\infty$ (since $\nabla P \in F \subset\{g<\infty\}$ a.e.).

In case $\Omega=\mathbb{R}^{N}$, one easily checks that (3.1) and (3.2) each hold if and only if $0 \in(\operatorname{co} F)^{\circ}$ (or, equivalently, $0 \in(\operatorname{co}\{g<\infty\})^{\circ}$, with a suitable choice of $\left.F \subset\{g<\infty\}\right)$. If $\Omega$ is not the whole space, it may happen that (3.1) or (3.2) leads to a weaker requirement on the set $\{g<\infty\}$. Our results below, including the ones of Section 4 , stay true if instead of $0 \in(\operatorname{co}\{g<\infty\})^{\circ}$, we assume that either (3.1) or (3.2) holds for a suitable finite set $F \subset\{g<\infty\}$. Some examples are indicated in Section 5 .

Proof of Lemma 3.5. The definition of $P$ immediately implies that $P$ is continuous, $P(0)=0$ and $P \in W_{\text {loc }}^{1, \infty}$ with $\nabla P \in F$ a.e. Moreover, for any $x \in \mathbb{R}^{N} \backslash\{0\}$, there exists a $\eta=\eta(x) \in F$ such that $x \cdot \eta<0$, because otherwise $F$ would be a subset of the half-space $\left\{y \in \mathbb{R}^{N} \mid x \cdot y \geq 0\right\}$, which contradicts our assumption that $0 \in(\operatorname{co} F)^{\circ}$. Hence $P(x)<0$ for every $x \neq 0$. Finally, since $P$ is 1 -homogeneous, this also implies that $P(x) \leq-a|x|$.

In particular, Lemma 3.5 leads to the following conditions to rule out trivial functionals. In all of them, we have to assume the existence of one function $u$ at which $G$ is finite. For the most general statement, we also need some control of the behavior of $u$ as $|x| \rightarrow \infty$, that is, we require "sublinear growth" at infinity in the sense that

$$
\{x \in \Omega|| u(x)|\geq a| x \mid\} \text { has finite measure for every } a>0 \text {. }
$$

Theorem 3.8. Suppose that there exists $u \in L^{1, p}(\Omega)$ with "sublinear growth" in the sense of (3.3) such that $G(u) \in \mathbb{R}$, and suppose that $0 \in(\operatorname{co}\{g<\infty\})^{\circ}$. Then $G$ is nontrivial on $L_{\sim}^{1, p}(\Omega)$. If, in addition, $u \in L^{q}(\Omega)$, then $G$ is also nontrivial on $L^{1, p} \cap L^{q}(\Omega)$.

The proof employs the following simple observation:

Lemma 3.9. Let $Q$ be a function in $L^{1, \infty}(\Omega)$ such that $Q(x) \leq-s|x|$ on $\Omega$, and suppose $u \in L^{1, p}(\Omega)$ satisfies (3.3). Then $\{u<Q\}=\{x \in \Omega \mid u(x)<Q(x)\}$ has finite measure and $v(x):=\max \{u(x), Q(x)\}$ also is a function in $L^{1, p}(\Omega)$, with $\nabla v=\nabla u$ a.e. on $\{u \geq Q\}$ and $\nabla v=\nabla Q$ a.e. on $\{u<Q\}$.

Proof. Certainly, $v$ is weakly differentiable, and its gradient is a function in $L_{\mathrm{loc}}^{p}$ of the form stated above. Moreover, since $Q(x) \leq-s|x|,(3.3)$ implies that $\{u<Q\}$ has finite measure. This, together with the fact that $\nabla Q$ is bounded, in turn entails that $\int_{\Omega}|\nabla v|^{p}<\infty$. 
Proof of Theorem 3.8. Choose a suitable finite subset $F \subset\{g<\infty\}$ such $0 \in(\operatorname{co} F)^{\circ}$, and let $P$ denote the associated pyramid introduced in Lemma 3.5. Then by the previous lemma, for any $h \in \mathbb{R}$,

$$
v_{h}:=\max \{u, P+h\}=h+\max \{u-h, P\}
$$

is a function in $L^{1, p}(\Omega)$. Moreover, $\left\{v_{h} \neq u\right\}$ is a set of finite measure and $(g \circ \nabla P)(\Omega) \subset \mathbb{R}$ is bounded, whence $G\left(v_{h}\right) \in \mathbb{R}$. If $u \in L^{q}(\Omega)$, we also get that $v_{h} \in L^{q}(\Omega)$. Finally, observe that $\left\{\left[v_{h}\right] \mid h \in \mathbb{R}\right\} \subset L_{\sim}^{1, p}(\Omega)$ is an infinite set, whence $G$ is nontrivial on $L_{\sim}^{1, p}(\Omega)$.

Observe that (3.3) holds for every $u \in\left(L^{1, p} \cap L^{q}\right)(\Omega)$, and (3.3) is not a very strong restriction for functions in $L^{1, p}$, either. In particular, Corollary A.4 in the appendix states that for a large class of domains, every function in $L^{1, p}$ satisfies (3.3).

Corollary 3.10. Suppose that $\Omega \subset \mathbb{R}^{N}$ has the properties required in Corollary A.4. Moreover, suppose that there exists $u \in L^{1, p}(\Omega)$ such that $G(u) \in \mathbb{R}$, and suppose that $0 \in(\operatorname{co}\{g<\infty\})^{\circ}$. Then $G$ is nontrivial on $L_{\sim}^{1, p}(\Omega)$.

Corollary 3.11. Suppose that there exists $u \in\left(L^{1, p} \cap L^{q}\right)(\Omega)$ such that $G(u) \in \mathbb{R}$, and suppose that $0 \in(\operatorname{co}\{g<\infty\})^{\circ}$. Then $G$ is nontrivial on $\left(L^{1, p} \cap L^{q}\right)(\Omega)$.

As already mentioned in Remark 3.7, $0 \in(\operatorname{co}\{g<\infty\})^{\circ}$ in general is not necessary for nontrivial $G$. However, this is the only condition on $g$ which guarantees a nontrivial functional independently of the domain, as it is necessary in case $\Omega=\mathbb{R}^{N}$. In particular, we have the following:

Corollary 3.12 $\left(\Omega=\mathbb{R}^{N}\right)$. G is nontrivial on $\left(L^{1, p} \cap L^{q}\right)\left(\mathbb{R}^{N}\right)$ if and only if there exists $u \in\left(L^{1, p} \cap L^{q}\right)\left(\mathbb{R}^{N}\right) \backslash\{0\}$ such that $G(u) \in \mathbb{R}$. Moreover, if $1<p<N$, the above remains true for $L_{\sim}^{1, p}\left(\mathbb{R}^{N}\right)$ instead of $\left(L^{1, p} \cap L^{q}\right)\left(\mathbb{R}^{N}\right)$.

Proof. As "only if" is trivial, it suffices to show the converse. The existence of $u \neq \equiv 0$ with $G(u) \in \mathbb{R}$ implies $0 \in(\operatorname{co}\{g<\infty\})^{\circ}$ by Example 3.4. Thus, the assertion is a consequence of the previous corollaries.

\section{NECESSARY CONDITIONS}

Our main results are the following:

Theorem 4.1. Suppose that $G(u) \in \mathbb{R}$ for a function $u \in L^{1, p}(\Omega)$ with "sublinear growth" in the sense of (3.3), and suppose that $0 \in(\operatorname{co}\{g \in \mathbb{R}\})^{\circ}$. Moreover, assume that $G$ is wslsc in $L_{\sim}^{1, p}(\Omega)$. Then $g$ is lsc and convex. If, in addition, the measure of $\Omega$ is infinite, then we also have that $g(0)=0$, and for every $\xi \in \mathbb{R}^{N}$,

$$
\begin{array}{ll}
g(\xi) \geq 0 \quad \text { if } 1<p<\infty \\
g(\xi) \geq \nu \cdot \xi \quad \text { if } p=1, \text { where } \nu \in \mathbb{R}^{N} \text { is a constant. }
\end{array}
$$

Theorem 4.2. Suppose that $G(u) \in \mathbb{R}$ for a function $u \in\left(L^{1, p} \cap L^{q}\right)(\Omega)$, and suppose that $0 \in(\operatorname{co}\{g \in \mathbb{R}\})^{\circ}$. Moreover, assume that $G$ is wslsc in $\left(L^{1, p} \cap L^{q}\right)(\Omega)$. Then the conclusion of Theorem 4.1 holds.

The proofs of the theorems are given at the end of this section.

Remark 4.3. Theorem 4.1 holds even for $p=\infty$ with only minor modifications in the proof (in the propositions below), if weak convergence throughout is replaced by weak*-convergence. In particular, $G$ is then assumed to be $\mathrm{w}^{*}-\operatorname{slsc}^{6}$ in $L_{\sim}^{1, \infty}(\Omega)$. The same can be said for Theorem 4.2 if either $p=\infty$ or $q=\infty$ or both.

\footnotetext{
${ }^{6}$ Sequentially lower semicontinuous with respect to weak*-convergence.
} 
Obviously, assumption (3.3) for $u$ in Theorem 4.1 can be dropped if Corollary A.4 is applicable (in particular, $p<N)$ :

Corollary 4.4. Suppose that $p$ and $\Omega$ satisfy the requirements of Corollary A.4, that $G(u) \in \mathbb{R}$ for a function $u \in L^{1, p}(\Omega)$, and that $0 \in(\operatorname{co}\{g \in \mathbb{R}\})^{\circ}$. Moreover, assume that $G$ is wslsc in $L_{\sim}^{1, p}(\Omega)$. Then the conclusion of Theorem 4.1 holds.

Finally, using Example 3.4 and Corollary 4.4, we observe that the simplest possible result holds for $\Omega=\mathbb{R}^{N}$ if $p<N$ :

Corollary $4.5\left(\Omega=\mathbb{R}^{N}\right)$. Suppose that $1<p<N$ and suppose that $G(u) \in \mathbb{R}$ for a function $u \in L^{1, p}\left(\mathbb{R}^{N}\right) \backslash\{0\}$. Moreover, assume that $G$ is wslsc in $L_{\sim}^{1, p}\left(\mathbb{R}^{N}\right)$. Then the conclusion of Theorem 4.1 holds.

Below, we repeatedly use the following notion of convergence of sets:

Definition 4.6 (convergence of sets in measure). Given a sequence $A_{n}$ of measurable sets in $\mathbb{R}^{N}$ and $A \subset \mathbb{R}^{N}$ measurable, we say that $A_{n} \rightarrow A$ in measure, or $\mathcal{L}^{N}-\lim A_{n}=A$, if

$$
\mathcal{L}^{N}\left(A \backslash A_{n}\right)+\mathcal{L}^{N}\left(A_{n} \backslash A\right) \rightarrow 0 .
$$

Here, $\mathcal{L}^{N}$ is the Lebesgue measure in $\mathbb{R}^{N}$.

The main part of the proof of Theorem 4.1 is split into two propositions.

Proposition 4.7. Suppose that $G(u) \in \mathbb{R}$ for a $u \in L^{1, p}(\Omega)$ with "sublinear growth" in the sense of (3.3), and suppose that $0 \in(\operatorname{co}\{g \in \mathbb{R}\})^{\circ}$. Moreover, assume that $G$ is strongly lsc in $L_{\sim}^{1, p}(\Omega)$. Then $g$ is lsc on $\mathbb{R}^{N}$.

Proof. It is enough to show that $g$ is lsc at every point $\xi \in \overline{\{g \in \mathbb{R}\}}$. Choose a finite subset $F$ of $\{g \in \mathbb{R}\}$ such that $0 \in(\operatorname{co} F)^{\circ}$, and let $P$ denote the associated pyramid introduced in Lemma 3.5. Moreover, let $\left(\xi_{n}\right) \subset \mathbb{R}^{N}$ be a sequence converging to $\xi$. W.l.o.g., we may assume that $g\left(\xi_{n}\right)$ is bounded in $\mathbb{R}$ (using (1.4) to obtain the bound from below). By extracting a subsequence, we can make sure that $\lim \inf g\left(\xi_{n}\right)$ is actually a limit. Thus, it suffices to show that

$$
\lim g\left(\xi_{n}\right) \geq g(\xi)
$$

To exploit the strong lsc of $G$, we construct a suitable sequence of functions $w_{n}$ having slope $\xi_{n}$ one a suitable sets of positive measure, converging strongly to a limit function $w$, in such a way that - roughly speaking the affine parts of $w_{n}$ converge to an affine part of $w$ with slope $\xi$ (Fig. 1). Before we give the details, let us describe the underlying idea of this construction: we first cut off the tip of $P$ (together with a whole side, if $\xi_{n}$ or $\xi$ does not lie in $(\operatorname{co} F)^{\circ}$ ) with an affine function of slope $\xi_{n}$ (or $\xi$ ), shift the result so that the former position of the tip moves to some $z \in \Omega$ and then take the maximum with $u$ to correct the behavior of this function for large $|x|$ to ensure that the resulting map $w_{n}$ (or $w$ ) belongs to $L^{1, p}(\Omega$ ). By first adding a suitably large constant $h$ to the truncated pyramid $Q_{n}$ (or $Q$ ), this can be done in such a way that a large part with slope $\xi_{n}$ (or $\xi$ ) is present in $w_{n}$ (or $w$ ). Technical difficulties arise mainly from the fact that we do not know anything about $u$ apart from (3.3), which makes controlling the unwanted side effects of the construction (caused by the set where $\nabla w=\xi$ but $\nabla w_{n} \neq \xi_{n}$ and vice versa) rather arduous. In particular, the arguments below could be greatly simplified if $u \equiv 0$.

For every $x \in \Omega$ and for the parameters $h \in \mathbb{R}$ (to be chosen later) and $z \in \Omega$ (chosen arbitrarily), let

$$
\begin{aligned}
& w(x):=\max \{u(x), Q(x-z)+h\} \quad \text { with } Q(y):=\min \{P(y), \xi \cdot y-1\}, \\
& w_{n}(x):=\max \left\{u(x), Q_{n}(x-z)+h\right\} \quad \text { with } Q_{n}(y):=\min \left\{P(y), \xi_{n} \cdot y-1\right\},
\end{aligned}
$$

and

$$
\begin{aligned}
& S:=\{x \in \Omega \mid P(x-z)>Q(x-z)\}, \quad S_{n}:=\left\{x \in \Omega \mid P(x-z)>Q_{n}(x-z)\right\} \\
& T:=\{x \in \Omega \mid u(x) \leq P(x-z)+h\}, \quad R:=\{x \in \Omega \mid u(x)=Q(x-z)+h\} .
\end{aligned}
$$




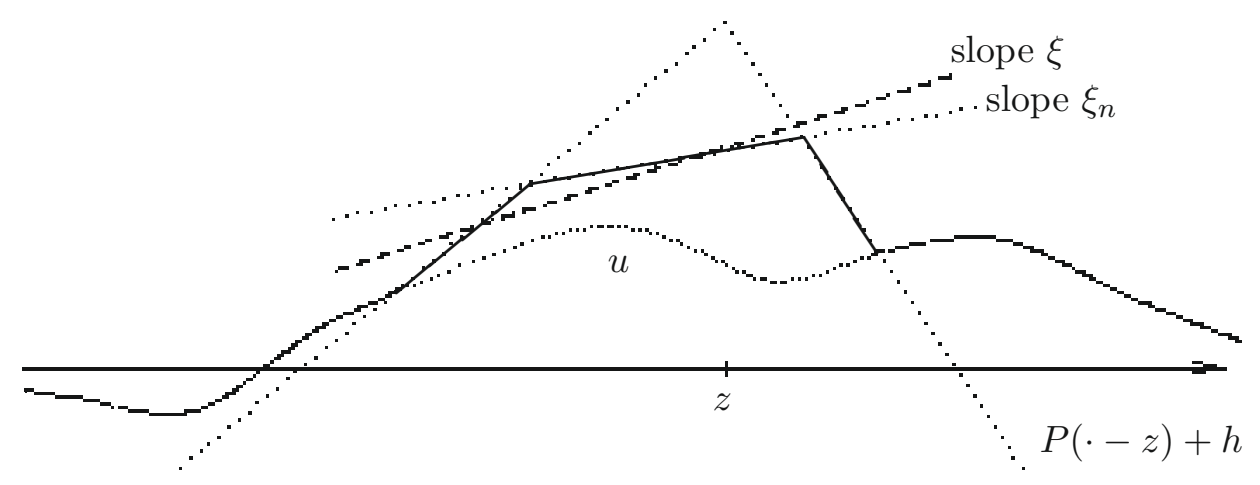

Figure 1. An example for $w_{n}$ with $\Omega=\mathbb{R}$, in case $\xi \in(\operatorname{co} F)^{\circ}$.

By definition,

$$
\begin{aligned}
& w=u=w_{n} \text { a.e. on } \Omega \backslash T \text { and thus } \nabla w=\nabla u=\nabla w_{n} \text { a.e. on } \Omega \backslash T, \\
& \text { and } T \text { is a set of finite measure (for fixed } h \text { ), }
\end{aligned}
$$

due to Lemma 3.5 and the "sublinear growth" (3.3) of $u$. By Lemma 3.9, $w \in L^{1, p}(\Omega), w_{n} \in L^{1, p}(\Omega)$ and $g\left(\nabla w_{n}\right) \in L^{1}(\Omega)$. To show the strong convergence of $w_{n}$ to $w$ in $L^{1, p}$, observe that in addition to (4.3), $\nabla w(x) \in\{\nabla u(x)\} \cup\{\xi\} \cup F$ on $T$ and $\nabla w_{n}(x) \in\{\nabla u(x)\} \cup\left\{\xi_{n}\right\} \cup F$ on $T$. Since $F \cup\{\xi\} \cup\left\{\xi_{n} \mid n \in \mathbb{N}\right\}$ is bounded in $\mathbb{R}$ and $\nabla w_{n} \rightarrow \nabla w$ pointwise a.e. (because $\nabla Q_{n} \rightarrow \nabla Q$ pointwise a.e.), this implies that $w_{n} \rightarrow w$ strongly in $L_{\sim}^{1, p}(\Omega)$ by dominated convergence. The same argument also shows that $g\left(\nabla w_{n}\right)$ is bounded in $L^{1}(\Omega)$, and in particular, we also have $g(\nabla w) \in L^{1}(\Omega)$ due to the strong lsc of $G$. By choosing $h$ sufficiently large, we can make sure that

$$
\mathcal{L}^{N}(T \cap S \cap\{u<w\}) \geq \frac{1}{2} \min \left\{\mathcal{L}^{N}(\Omega \cap S), 1\right\}>0,
$$

since the measure of $\{u<w\} \cap S \subset T$ becomes arbitrarily large as $h \rightarrow \infty$.

We are now in position to exploit the strong lsc of $G$. In the following, let $V$ denote any measurable set with

$$
S \cap\{u<w\} \subset V \subset S \cap(\{u<w\} \cup R) .
$$

As a consequence, $\nabla w=\xi$ a.e. on $V$ by definition of $S$ and $R$, and we have

$$
\begin{aligned}
\int_{V} g(\xi)+\int_{T \backslash S} g(\nabla w) & +\int_{(T \cap S) \backslash V} g(\nabla u)=G(w)-\int_{\Omega \backslash T} g(\nabla u) \\
& \leq \liminf G\left(w_{n}\right)-\int_{\Omega \backslash T} g(\nabla u) \\
& =\liminf \left(\int_{T \cap S_{n} \cap\left\{u<w_{n}\right\}} g\left(\xi_{n}\right)+\int_{T \backslash S_{n}} g(\nabla w)+\int_{\left(T \cap S_{n}\right) \backslash\left\{u<w_{n}\right\}} g(\nabla u)\right)
\end{aligned}
$$

where we used (4.3) and (4.5). It remains to resolve the liminf in the last line of (4.6). We claim that $V$ can be chosen in such a way that

$$
\begin{aligned}
\liminf \left(\int_{T \cap S_{n} \cap\left\{u<w_{n}\right\}} g\left(\xi_{n}\right)+\int_{T \backslash S_{n}} g(\nabla w)+\int_{\left(T \cap S_{n}\right) \backslash\left\{u<w_{n}\right\}} g(\nabla u)\right)= & \int_{V} \lim g\left(\xi_{n}\right) \\
& +\int_{T \backslash S} g(\nabla w)+\int_{T \backslash V} g(\nabla u)
\end{aligned}
$$


holds in addition to (4.5), at least for a suitable subsequence of $\xi_{n}$ (not relabeled). Postponing the proof of this for a moment, observe that (4.6) and (4.7) imply that

$$
\mathcal{L}^{N}(V) g(\xi)=\int_{V} g(\xi) \leq \lim \int_{V} g\left(\xi_{n}\right)=\mathcal{L}^{N}(V) \lim g\left(\xi_{n}\right),
$$

and this yields (4.2), since $0<\mathcal{L}^{N}(V) \leq \mathcal{L}^{N}(T)<\infty$ due to (4.4).

The proof of (4.7) is essentially a consequence of Lebesgue's theorem on dominated convergence and the regularity of the Lebesgue measure. First, we claim that

$$
M \cap S_{n} \rightarrow M \cap S \text { in measure for every } M \subset \Omega \text { with finite measure. }
$$

For a proof, note that due to the locally uniform convergence of $Q_{n}$ to $Q$,

$$
\left.\mathcal{L}^{N}\left(M \cap\left(S \backslash S_{n}\right)\right) \rightarrow 0 \text { and } \mathcal{L}^{N}\left(M \cap\left(S_{n} \backslash \tilde{S}\right)\right]\right) \rightarrow 0,
$$

where $\tilde{S}:=\{x \in \Omega \mid P(x-z) \geq Q(x-z)\}$. This already yields (4.8) since $\tilde{S} \backslash S=\{x \in \Omega \mid P(x-z)=Q(x-z)\}$ is a set of measure zero, due to the fact that the affine function $f(x):=x \cdot \xi-1$ always intersects $P$ transversally (this is obvious if $\xi \notin F \supset \nabla P\left(\mathbb{R}^{N}\right)$, and even if $\xi \in F, \nabla P(\tilde{S} \backslash S) \subset F \backslash\{\xi\}$ as the side of $P$ with slope $\xi$ gets cut off completely by $f$ in the definition of $Q$ ). As an immediate consequence of (4.8), also using that $g(\nabla u) \in L^{1}(\Omega), g(\nabla w) \in L^{1}(\Omega)$ and that $g\left(\xi_{n}\right)$ is bounded, we may replace $S_{n}$ with $S$ or $S \cap S_{n}$ in (4.7) and it thus suffices to show that

$$
\begin{aligned}
& \lim \int_{T \cap S \cap S_{n} \cap\left\{u<w_{n}\right\}} g\left(\xi_{n}\right)=\int_{T \cap S \cap V} g(\xi), \\
& \lim \int_{\left(T \cap S \cap S_{n}\right) \backslash\left\{u<w_{n}\right\}} g(\nabla u)=\int_{(T \cap S) \backslash V} g(\nabla u) .
\end{aligned}
$$

Since $g\left(\xi_{n}\right) \rightarrow g(\xi)$ and $g(\nabla u) \in L^{1}(\Omega),(4.9)$ in turn follows once we show that

$$
T \cap S \cap S_{n} \cap\left\{u<w_{n}\right\}=T \cap S \cap S_{n} \cap\left\{u<Q_{n}(\cdot-z)+h\right\} \rightarrow V \text { in measure }
$$

for a suitable $V$. As a first step, observe that

$$
T \cap S \cap S_{n} \cap\left\{u<w_{n}\right\} \cap\{u<w\} \rightarrow T \cap S \cap\{u<w\} \text { in measure, }
$$

since $w_{n} \rightarrow w$ pointwise a.e. and $T$ has finite measure. Moreover, since $u>Q(\cdot-z)+h$ on $\Omega \backslash(R \cup\{u<w\})$, the same argument yields that

$$
\left(T \cap S \cap S_{n} \cap\left\{u<Q_{n}(\cdot-z)+h\right\}\right) \backslash(R \cup\{u<w\}) \rightarrow \emptyset \text { in measure. }
$$

To discuss the remainder, the limit of $T \cap S \cap S_{n} \cap\left\{u<Q_{n}(\cdot-z)+h\right\} \cap R$, we have to distinguish the points where $Q_{n}>Q$ or $Q<Q_{n}$, respectively, and since we work on $S \cap S_{n}$, this comes down to comparing the affine functions $x \mapsto(x-z) \cdot \xi$ and $x \mapsto(x-z) \cdot \xi_{n}$. By passing to a subsequence of $\xi_{n}$ (not relabeled), we may assume that

$$
\frac{\xi-\xi_{n}}{\left|\xi-\xi_{n}\right|} \underset{n \rightarrow \infty}{\longrightarrow} \eta, \text { for an } \eta \in \mathbb{R}^{N} \text { with }|\eta|=1
$$

Define

$$
H_{n}:=\left\{x \in \Omega \mid(x-z) \cdot \frac{\xi-\xi_{n}}{\left|\xi-\xi_{n}\right|}<0\right\}, \quad H:=\{x \in \Omega \mid(x-z) \cdot \eta<0\}
$$


and observe that

$$
T \cap H_{n} \rightarrow T \cap H \text { in measure }
$$

due to (4.13). Together with (4.8), this implies that

$$
T \cap S \cap S_{n} \cap\left\{u<Q_{n}(\cdot-z)+h\right\} \cap R=T \cap S \cap S_{n} \cap H_{n} \cap R \rightarrow T \cap S \cap H \cap R .
$$

Combined, (4.11), (4.12) and (4.14) yield (4.10) for

$$
V:=[T \cap S \cap\{u<w\}] \cup[T \cap S \cap R \cap H]
$$

which obviously satisfies (4.5). This concludes the proof of (4.7).

In case $G$ is wslsc, we obtain:

Proposition 4.8. Suppose that $G(u) \in \mathbb{R}$ for a $u \in L^{1, p}(\Omega)$ with "sublinear growth" in the sense of (3.3), and suppose that $0 \in(\operatorname{co}\{g \in \mathbb{R}\})^{\circ}$. Moreover, assume that $G$ is wslsc in $L_{\sim}^{1, p}(\Omega)$. Then $g$ is convex on $\mathbb{R}^{N}$.

Proof. Let $\xi_{1}, \xi_{2} \in \mathbb{R}^{N}$ with $\xi_{1} \neq \xi_{2}$ such that $g\left(\xi_{1}\right)<\infty$ and $g\left(\xi_{2}\right)<\infty$. We have to show that for every $\theta_{1}, \theta_{2} \in(0,1)$ with $\theta_{1}+\theta_{2}=1$,

$$
g(\bar{\xi}) \leq \theta_{1} g\left(\xi_{1}\right)+\theta_{2} g\left(\xi_{2}\right), \text { where } \bar{\xi}:=\theta_{1} \xi_{1}+\theta_{2} \xi_{2}
$$

Let $F$ be a finite subset of $\operatorname{co}\{g \in \mathbb{R}\}$ such that $0 \in(\operatorname{co} F)^{\circ}$ and let $P$ denote the associated pyramid introduced in Lemma 3.5. We are going to prove (4.15) with arguments very similar to those applied in Proposition 4.7. Essentially, we now truncate the pyramid with a laminate $\lambda_{n}$ composed of piecewise affine functions whose gradient oscillates faster and faster in $\left\{\xi_{1}, \xi_{2}\right\}$ with average slope $\bar{\xi}$. For any $n \in \mathbb{N}$ and $y \in \mathbb{R}^{N}$ define

$$
\begin{aligned}
\lambda(y):=\bar{\xi} \cdot y \text { and } \lambda_{n}(y):= & \lambda(y)+\max \left\{\Lambda\left(y+2^{-n} k\left(\xi_{1}-\bar{\xi}\right)\right) \mid k \in \mathbb{Z}\right\}, \\
& \text { where } \Lambda(y):=\min \left\{\left(\xi_{1}-\bar{\xi}\right) \cdot y,\left(\xi_{2}-\bar{\xi}\right) \cdot y\right\} .
\end{aligned}
$$

Observe that $\lambda, \lambda_{n} \in W_{\text {loc }}^{1, \infty}\left(\mathbb{R}^{N}\right)$,

$$
\begin{aligned}
& \lambda \in W_{\text {loc }}^{1, \infty}\left(\mathbb{R}^{N}\right), \lambda_{n} \in W_{\text {loc }}^{1, \infty}\left(\mathbb{R}^{N}\right), \lambda_{n} \leq \lambda_{n+1} \leq \lambda, \nabla \lambda_{n} \in\left\{\xi_{1}, \xi_{2}\right\} \text { a.e. } \\
& \lambda_{n}-\lambda \rightarrow 0 \text { in } L^{\infty}\left(\mathbb{R}^{N}\right), \text { and } \nabla \lambda_{n} \rightarrow^{*} \nabla \lambda=\bar{\xi} \text { in } L^{\infty}\left(\mathbb{R}^{N}\right) .
\end{aligned}
$$

Moreover,

$$
\mathcal{L}^{N}\left(M \cap\left\{\nabla \lambda_{n}=\xi_{i}\right\}\right) \underset{n \rightarrow \infty}{\longrightarrow} \theta_{i} \mathcal{L}^{N}(M), \quad i=1,2
$$

for every fixed measurable set $M \subset \mathbb{R}^{N}$ with finite measure. The laminate can be built into admissible functions for $G$ just as in Proposition 4.7. For every $x \in \Omega$ and for parameters $h \in \mathbb{R}$ (chosen below) and $z \in \Omega$ (chosen arbitrarily), let

$$
\begin{aligned}
& w(x):=\max \{u(x), Q(x-z)+h\} \quad \text { with } Q(y):=\min \{P(y), \lambda(y)-1\} \\
& w_{n}(x):=\max \left\{u(x), Q_{n}(x-z)+h\right\} \quad \text { with } Q_{n}(y):=\min \left\{P(y), \lambda_{n}(y)-1\right\}
\end{aligned}
$$

and

$$
\begin{aligned}
& S:=\{x \in \Omega \mid P(x-z)>Q(x-z)\}, S_{n}:=\left\{x \in \Omega \mid P(x-z)>Q_{n}(x-z)\right\} \\
& T:=\{x \in \Omega \mid u(x)<P(x-z)+h\} .
\end{aligned}
$$


We now list a few consequences of these definitions which will be used later. First, note that

$$
\begin{aligned}
& w=w_{n}=u \text { on } \Omega \backslash T \text { and thus } \nabla w=\nabla w_{n}=\nabla u \text { a.e. on } \Omega \backslash T, \\
& \text { and } T \text { is a set of finite measure (for fixed } h \text { ), }
\end{aligned}
$$

due to Lemma 3.5 and the "sublinear growth" (3.3) of $u$. In particular, $w, w_{n} \in L_{\sim}^{1, p}(\Omega)$. Second, since $\lambda \geq \lambda_{n+1} \geq \lambda_{n}$ on $\mathbb{R}^{N}$ in $L^{\infty}\left(\mathbb{R}^{N}\right)$, we also have

$$
w \geq w_{n+1} \geq w_{n}, \text { and } S \subset S_{n+1} \subset S_{n} \text { for all } n \in \mathbb{N},
$$

properties we could not get in Proposition 4.7, and which will allow us to use $V:=T \cap S \cap\{u<w\}$. Third,

$$
M \cap S_{n} \rightarrow M \cap S \text { in measure for every } M \subset \Omega \text { with finite measure, }
$$

which can be shown in the same way as (4.8) in the proof of Proposition 4.7. As a consequence of (4.20), $\nabla w_{n} \rightarrow \nabla w$ strongly in $L^{p}\left(T \backslash S ; \mathbb{R}^{N}\right.$ ) (note that $w_{n}=w$ on $\Omega \backslash\left(S_{n} \cup S\right.$ ), and the sequence $\left|\nabla w_{n}\right|^{p}$ is equiintegrable by construction). In addition, we have that $\nabla w_{n}=\nabla \lambda_{n} \rightarrow \nabla \lambda=\nabla w$ weakly in $L^{p}\left(T \cap S ; \mathbb{R}^{N}\right)$. Since $w_{n}=u=w$ on $\Omega \backslash T$, we infer that $\nabla w_{n} \rightarrow \nabla w$ weakly in $L^{p}\left(\Omega ; \mathbb{R}^{N}\right)$, or, equivalently, $w_{n} \rightarrow w$ weakly in $L_{\sim}^{1, p}(\Omega)$, so that we may employ the wslsc of $G$ along the sequence $w_{n}$. In particular, $g(\nabla w) \in L^{1}(\Omega)$ as $G\left(w_{n}\right)$ is bounded. As in the proof of Proposition 4.7, we choose $h$ large enough so that

$$
\mathcal{L}^{N}(T \cap S \cap\{u<w\}) \geq \frac{1}{2} \min \left\{\mathcal{L}^{N}(\Omega \cap S), 1\right\}>0 .
$$

Due to the wslsc of $G$, we have

$$
\begin{aligned}
\mathcal{L}^{N}(T \cap S \cap\{u<w\}) g(\xi)+ & \int_{T \backslash S} g(\nabla w)+\int_{T \cap S \cap\{u \geq w\}} g(\nabla u)=G(w)-\int_{\Omega \backslash T} g(\nabla u) \\
\leq & \liminf G\left(w_{n}\right)-\int_{\Omega \backslash T} g(\nabla u) \\
= & \liminf \left(\int_{T \cap S_{n} \cap\left\{u<w_{n}\right\}} g\left(\nabla \lambda_{n}\right)+\int_{T \backslash S_{n}} g(\nabla w)+\int_{T \cap S_{n} \cap\left\{u \geq w_{n}\right\}} g(\nabla u)\right) \\
= & \liminf \left(\sum_{i=1}^{2} \mathcal{L}^{N}\left(T \cap S_{n} \cap\left\{u<w_{n}\right\} \cap\left\{\nabla \lambda_{n}=\xi_{i}\right\}\right) g\left(\xi_{i}\right)\right. \\
& \left.+\int_{T \backslash S_{n}} g(\nabla w)+\int_{T \cap S \cap\{u \geq w\}} g(\nabla u)\right) \\
= & \mathcal{L}^{N}(T \cap S \cap\{u<w\})\left(\theta_{1} g\left(\xi_{1}\right)+\theta_{2} g\left(\xi_{2}\right)\right)+\int_{T \backslash S} g(\nabla w)+\int_{T \cap S \cap\{u \geq w\}} g(\nabla u),
\end{aligned}
$$

where we used (4.3) and (4.5), and the last equality above still has to be justified. Postponing this for a moment, note that as a consequence,

$$
A g(\xi) \leq A\left(\sigma_{1} g\left(\xi_{1}\right)+\sigma_{2} g\left(\xi_{2}\right)\right), \text { where } A:=\mathcal{L}^{N}(T \cap S \cap\{u<w\})
$$

which implies (4.15). Here, note that $A$ is finite and bounded away from zero due to (4.21).

As a consequence of Lebesgue's theorem on dominated convergence, (4.20) and the fact that $g(\nabla w) \in L^{1}(\Omega)$, the last equality in (4.22) can be checked by showing that

$$
T \cap S_{n} \cap\left\{u<w_{n}\right\} \rightarrow T \cap S \cap\{u<w\} \text { in measure }
$$


and

$$
\mathcal{L}^{N}\left(T \cap S_{n} \cap\left\{u<w_{n}\right\} \cap\left\{\nabla \lambda_{n}=\xi_{i}\right\}\right) \underset{n \rightarrow \infty}{\longrightarrow} \mathcal{L}^{N}(T \cap S \cap\{u<w\}) \theta_{i}
$$

for $i=1,2$. Here, we may replace $S_{n}$ with $S$ due to (4.20), and we also may replace $\left\{u<w_{n}\right\}$ by $\{u<w\}$, since $\left\{u<w_{n}\right\} \subset\{u<w\}$ due to (4.19) and

$$
\mathcal{L}^{N}\left(\{u<w\} \backslash\left\{u<w_{n}\right\}\right) \rightarrow 0,
$$

where we used that $w-w_{n} \rightarrow 0$ pointwise in $\Omega$ and $\{u<w\} \subset T$ is a set of finite measure. This already finishes the proof of (4.23), and (4.24) is a consequence of (4.23) combined with (4.17).

Proof of Theorem 4.1. Since wslsc of $G$ implies strong lsc of $G$, lsc of $g$ is a consequence of Proposition 4.7 below, and convexity of $g$ is due to Proposition 4.8. Now suppose that $\mathcal{L}^{N}(\Omega)=\infty$. In this case, the existence of $u$ with $\int_{\Omega}|g(\nabla u)|<\infty$ and $\int_{\Omega}|\nabla u|^{p}<\infty$ implies that there is a sequence $\xi_{n} \rightarrow 0$ such that $g\left(\xi_{n}\right) \rightarrow 0$. Since $g$ is lsc and (1.4) holds, we infer that $g(0)=0$. If $p>1$, any convex function satisfying (1.4) and $g(0)=0$ has to be nonnegative. Finally, if $p=1$, the subdifferential of the convex function $g$ at 0 contains at least one point $\nu \in \mathbb{R}^{N}$, and since $g(0)=0$ and 0 is contained in the interior of $\{g<\infty\}=\operatorname{co}\{g \in \mathbb{R}\}$, this entails (4.1).

Proof of Theorem 4.2. First, observe that Proposition 4.7 and Proposition 4.8 remain valid if $L_{\sim}^{1, p}(\Omega)$ is replaced by $\left(L^{1, p} \cap L^{q}\right)(\Omega)$ and $u \in\left(L^{1, p} \cap L^{q}\right)(\Omega)$ is given (in particular, $u$ then satisfies (3.3)). In fact, the only thing we have to show in addition in the proofs of the propositions is that in both cases, the corresponding constructed sequence $w_{n}$ also satisfies $w_{n} \rightarrow w$ strongly in $L^{q}(\Omega)$ (in Prop. 4.8, weak convergence would actually suffice, but we get strong convergence anyway). Since $w_{n}=w=u$ on $\Omega \backslash T$ where $T$ is a set of finite measure, $w_{n} \rightarrow w$ in $L^{q}(\Omega)$ is easily shown using dominated convergence. The rest of the proof is analogous to the proof of Theorem 4.1.

\section{Concluding REMARKS}

It is remarkable that the proof presented here does not directly use the assumption that the functional is nontrivial. Instead, we had to assume that

$$
0 \in(\operatorname{co}\{g<\infty\})^{\circ} \subset \mathbb{R}^{N},
$$

which as we saw is sufficient for $G$ to be nontrivial on $\left(L^{1, p} \cap L^{q}\right)(\Omega)$ (if $G \not \equiv \infty$ ) with an arbitrary domain $\Omega$, but only for $\Omega=\mathbb{R}^{N}$, we know that (5.1) is necessary as well. It is actually not too difficult to find and use suitable weaker replacements for this assumption on other domains with simple geometry, which again are necessary and sufficient for nontrivial $G$. Basically, to get good candidates, we have to find characterizations of (3.1) and (3.2), for given $\Omega$ in terms of the set $\{g<\infty\}$. Of course, while each such characterization always yields a sufficient condition for nontrivial $G$, their necessity still remains unclear in general. Below, two simple examples are given.

Example 5.1. For infinite strips of the form

$$
\Omega=\mathbb{R}^{k} \times \omega \text { with a bounded domain } \omega \subset \mathbb{R}^{N-k},
$$

one can check that (3.1) and (3.2) each hold (with $P$ defined as in Lem. 3.5, associated to a suitable finite set $F \subset\{g<\infty\})$ if and only if

$$
0 \in\left(\operatorname{co} P_{k}(\{g<\infty\})\right)^{\circ} \subset \mathbb{R}^{k},
$$


where $P_{k}$ is the projection $\left(x_{1}, \ldots, x_{N}\right) \mapsto\left(x_{1}, \ldots, x_{k}\right)$. Accordingly, $G$ defined on $\left(L^{1, p} \cap L^{q}\right)\left(\mathbb{R}^{k} \times \omega\right)$ is nontrivial if and only if $G \not \equiv \infty$ and (5.2) is satisfied (here, note that Ex. 3.4 can be easily adapted to show "only if"). Moreover, (5.1) can be replaced by (5.2) in our main results for $\Omega=\mathbb{R}^{k} \times \omega$.

Example 5.2. For an infinite circular cone

$$
\Omega=\Lambda:=\left\{x \in \mathbb{R}^{N}|x \cdot e>\gamma| x \mid\right\}, \text { with } e \in S^{N-1} \text { and } \gamma \in[-1,1) \text { fixed, }
$$

one can check that (3.1) holds (with $P$ defined as in Lem. 3.5, associated to a suitable finite set $F \subset\{g<\infty\}$ ) if and only if

$$
\bar{\Lambda} \subset\{0\} \cup U \text {, where } U:=\bigcup_{\eta \in\{g<\infty\}}\left\{x \in \mathbb{R}^{N} \mid x \cdot \eta<0\right\},
$$

and consequently, (3.2) holds if an only if

$$
-\bar{\Lambda} \subset\{0\} \cup U
$$

Accordingly, $G$ defined on $\left(L^{1, p} \cap L^{q}\right)(\Lambda)$ is nontrivial if $G \not \equiv \infty$ and either (5.3) or (5.4) holds. If $\Lambda$ is a convex cone, i.e., if $\gamma \geq 0$, the converse is also true due to Example 3.3. Moreover, (5.1) can be replaced by (5.3) as well as by (5.4) in our main results for $\Omega=\Lambda$.

Already in Example 5.2 with $\gamma<0$, (5.3) or (5.4) combined with $G \not \equiv \infty$ is in fact no longer necessary for nontrivial $G$. Moreover, trying to find a reasonably general classification of domains with a corresponding list of characterizations for trivial functionals in terms of $g$ seems to be a pretty hopeless task. It thus would be interesting to know whether necessary conditions for wslsc of $G$ in $L_{\sim}^{1, p}$, especially if $L_{\sim}^{1, p}$ is not embedded into some $L^{q}$, can be obtained without using domain-dependent assumptions like (5.1)-(5.4) or their technical counterparts (3.1) and (3.2), respectively, instead trying to exploit directly that there is more than one function on which $G$ is known to be finite.

Another interesting question is whether our results, which are for scalar fields only, can be extended to the vector case. Technically, this would require fundamentally new ideas, since joining pieces of functions together by taking their maximum or minimum, which is the main method employed here, then no longer works well. Moreover, characterizing trivial functionals on $\mathbb{R}^{N}$ again becomes a challenging - and maybe related - task. We probably have to replace the convex hull in (5.1) by an appropriate notion of a quasiconvex hull. But which one exactly? And how to use it? Is there always a suitable replacement for the pyramid in this case?

\section{A. Appendix: Properties of $L^{1, p}(\Omega)$}

We recall embeddings of $L^{1, p}$ on unbounded domains originally obtained in [6] in a framework more general than presented here. For this purpose, we need certain properties of $\Omega$ specified below. The simplest examples for domains satisfying $(\Omega: 1)$ and $(\Omega: 2)$ below are the whole space and infinite (circular) cones.

\section{Definition A.1.}

(i) A cone (with vertex at 0 ) is a set of the form

$$
V=V(e, \varepsilon):=\left\{x \in \mathbb{R}^{N}|e \cdot x>(1-\varepsilon)| x \mid\right\},
$$

where $e \cdot x$ denotes the Euclidean scalar product. (The parameters $\varepsilon \in(0,1)$ and $e \in S^{N-1}$ specify the opening angle and the axis direction of the cone.)

(ii) Two domains $\Omega_{i}, \Omega_{j} \subset \mathbb{R}^{N}$ are said to overlap on an outer cone if there is a cone $V$ and a radius $R>0$ such that $V \backslash \bar{B}_{R}(0) \subset \Omega_{i} \cap \Omega_{j}$. 
(iii) $\Omega \subset \mathbb{R}^{N}$ satisfies the infinite cone condition, if there exists a cone $V$ such that $\Omega+V:=\{x+y \mid x \in$ $\Omega, y \in V\} \subset \Omega$.

(iv) $\Omega \subset \mathbb{R}^{N}$ satisfies condition $\left(\Omega: 1^{\prime}\right)$, if

$\Omega$ satisfies the infinite cone condition with a cone $V(e, \varepsilon)$, and there is a $\mu \in(0,1)$ such that $e \cdot x>\mu^{2}|x|$ for every $x \in \Omega_{i}$.

(v) $\Omega \subset \mathbb{R}^{N}$ satisfies condition $(\Omega: 1)$, if

$\Omega=\cup_{i=1}^{k} \Omega_{i}$ for a finite number $k$ of subdomains $\Omega_{i} \subset \Omega$,

where each $\Omega_{i}$ satisfies condition $\left(\Omega: 1^{\prime}\right)$ and for any $j \geq 2$,

there is an $i<j$ such that $\Omega_{i}$ and $\Omega_{j}$ overlap on an outer cone.

(This is condition (A) in [6].)

(vi) $\Omega \subset \mathbb{R}^{N}$ satisfies condition $\left(\Omega: 2^{\prime}\right)$, if

there exist $z \in \mathbb{R}^{N}$ and $R>0$ such that $\left[z+R \frac{x-z}{|x-z|}, x\right]$

is contained in $\Omega$ for every $x \in \Omega$ with $|x-z|>R$.

(vii) $\Omega \subset \mathbb{R}^{N}$ satisfies condition $(\Omega: 2)$, if

$\Omega=\cup_{i=1}^{k} \cup_{j=1}^{l_{i}} \Omega_{i j}$ for a finite number of subdomains $\Omega_{i j}$,

such that each $\Omega_{i j}$ satisfies condition $\left(\Omega: 2^{\prime}\right)$ and

$\tilde{\Omega}_{i}:=\cup_{j=1}^{l_{i}} \Omega_{i j}$ satisfies the infinite cone condition for every $i$.

(This is condition (B) in [6].)

If $p<N, L_{\sim}^{1, p}$ is continuously embedded in $L^{p^{*}}$ for domains satisfying $(\Omega: 1)$, as the following result shows.

Theorem A.2 (cf. Thm. 1 in [6]). Suppose that $1<p<N$ and that $\Omega \subset \mathbb{R}^{N}$ is a domain satisfying $(\Omega: 1)$. Then for any $u \in L^{1, p}(\Omega)$, there is a $h \in \mathbb{R}$ such that $u-h$ satisfies Sobolev's inequality in the form

$$
\left(\int_{\Omega}|u(x)-h|^{p^{*}} \mathrm{~d} x\right)^{\frac{1}{p^{*}}} \leq C\left(\int_{\Omega}|\nabla u|^{p} \mathrm{~d} x\right)^{\frac{1}{p}}
$$

with a constant $C=C(N, p, \Omega)>0$. Here, $p^{*}:=\frac{p N}{N-p}$.

If $p \geq N$, the result above (with $p^{*}=\infty$ ) is false in general. Nevertheless, something can still be said in form of an estimate for a weighted norm.

Theorem A.3 (cf. Thms. 2 and 3 in [6]). Suppose that $1<N \leq p<\infty$, that $\Omega \subset \mathbb{R}^{N}$ is a domain satisfying $(\Omega: 2)$ and and that $B \subset \Omega$ is a (bounded) open ball. Then for $q \in[p, \infty)$ and for any $u \in L^{1, p}(\Omega)$,

$$
\left(\int_{\Omega}(\gamma(x)|u(x)|)^{q} \mathrm{~d} x\right)^{\frac{1}{q}} \leq C\left(\int_{\Omega}|\nabla u|^{p} \mathrm{~d} x+\int_{B}|u|^{p} \mathrm{~d} x\right)^{\frac{1}{p}}
$$

with a constant $C=C(N, p, q, \Omega, B)>0$. Here, the weight $\gamma$ is given by

$$
\gamma(x):= \begin{cases}(1+|x|)^{-1+\frac{N}{p}-\frac{N}{q}} & \text { if } p>N, \\ (1+|x|)^{-1+\frac{N}{p}-\frac{N}{q}}(1+|\ln (|x|)|)^{-1} & \text { if } p=N>1 .\end{cases}
$$


Moreover, the above is also true for $p=\infty$, as well as for $q=\infty$ if $N<p$, where the corresponding integral norm in (A.2) has to be replaced by the essential supremum.

In some sense, this entails sublinear growth at infinity whenever $p<\infty$ :

Corollary A.4. Let $\Omega \subset \mathbb{R}^{N}$ be open and connected. Moreover, suppose that either $1<p<N$ and $\Omega$ is a finite union of subdomains $\Omega_{i}$ such that each $\Omega_{i}$ satisfies $\left(\Omega: 1^{\prime}\right)$ (but they do not necessarily overlap), or $1<N \leq p<\infty$ and $\Omega$ satisfies $(\Omega: 2)$. Then for any $u \in L^{1, p}(\Omega)$ and any $a>0$, the set

$$
\{|u|>a|\cdot|\}:=\{x \in \Omega|| u(x)|>a| x \mid\}
$$

has finite Lebesgue measure.

Proof. If $p<N$, since $\left(\Omega: 1^{\prime}\right)$ implies $(\Omega: 1)$, (A.1) holds on each $\Omega_{i}$, with possibly different numbers $h_{i}$. Of course, this is only possible if $\{|u|>a|\cdot|\} \cap \Omega_{i}$ has finite measure for each $i$. If $p \geq N$, the weight function on the left hand side of (A.2) satisfies $\gamma(x)|x| \rightarrow \infty$ as $|x| \rightarrow \infty$ for any choice of $q>p$, and thus the measure of $\{|u|>a|\cdot|\}$ is finite as a consequence o (A.2). Here, note that the right hand side of (A.2) is finite since $L^{1, p} \subset W_{\text {loc }}^{1, p}$ by definition.

Acknowledgements. I wish to thank Irene Fonseca, who suggested this topic. The financial support I received from the Deutsche Forschungsgemeinschaft (fellowship KR 3544/1-1), as well as the hospitality of the Center for Nonlinear Analysis (NSF Grants No. DMS-0405343 and DMS-0635983), are also gratefully acknowledged.

\section{REFERENCES}

[1] B. Dacorogna, Direct methods in the calculus of variations, Applied Mathematical Sciences 78. Springer, Berlin etc. (1989).

[2] I. Fonseca and G. Leoni, Modern Methods in the Calculus of Variations: L ${ }^{p}$ Spaces, Springer Monographs in Mathematics. Springer, New York (2007).

[3] E. Giusti, Direct methods in the calculus of variations. World Scientific, Singapore (2003).

[4] W. Gustin, On the interior of the convex hull of an Euclidean set. Bull. Am. Math. Soc. 53 (1947) $299-301$.

[5] V.G. Maz'ya, Sobolev spaces. Springer-Verlag, Berlin etc. (1985).

[6] Yu.S. Nikol'skij, Integral estimates for differentiable functions on unbounded domains. Proc. Steklov Inst. Math. 170 (1987) 267-283. Translation from Tr. Mat. Inst. Steklova 170 (1984) 233-247 (Russian). 